\title{
Primary Intestinal Hodgkin's Lymphoma: An Uncommon Presentation
}

\section{Shruti Sharma, Safia Rana', Sujala Kapur², Zeeba Shamim Jairajpuri ${ }^{1}$}

Department of CTVS (Cardiac pathology), All India Institute of Medical Sciences, Departments of Pathology, ${ }^{1}$ Hamdard Institute of Medical Sciences and Research, Jamia Hamdard, ${ }^{2}$ National Institute of Pathology, Indian Council of Medical Research, Safdarjung Hospital, New Delhi, India

Address for correspondence: Dr. Zeeba Shamim Jairajpuri, E-mail: zeebasj@rediffmail.com

\section{ABSTRACT}

Primary intestinal lymphoma is a rare lymphoproliferative neoplasm of the small intestine. The primary nature is established on the basis of lack of evidence of lymphoma on chest X-ray, computerized tomographic scan, peripheral blood or bone marrow puncture. Tumor involvement is limited to the gastrointestinal tract, the criteria for inclusion are that the symptoms related to the small intestine are predominant or the only symptoms at the time of laparotomy. Hodgkin's lymphoma $(\mathrm{HL})$ primarily in the small intestine is a rare entity and an uncommon presentation of the disease. Ileum is the more common site of infliction than the jejunum because of its abundant lymphoid follicles. Here, we present a case of primary intestinal $\mathrm{HL}$, in a 30 -year-old male.

Key words: Hodgkin's lymphoma, primary, small intestine

\section{INTRODUCTION}

Prim of rimary classical Hodgkin's lymphoma (HL) of the gastrointestinal tract (GIT) are rare lymphoproliferative neoplasm representing less than $5 \%$ of GIT lymphomas, predominantly confined to the small intestine. ${ }^{[1-3]}$ Diagnosis of the primary lesion requires the lack of peripheral or mediastinal lymphadenopathy, normal white blood cell (WBC) count and differential on peripheral blood smear. ${ }^{[4]}$ The most common site of extranodal lymphoma is the GIT with the majority of cases in the ileum due to the abundant lymphoid follicles. ${ }^{[5]}$ Pulmonary system, thyroid, skin, genitourinary and central nervous system being the other sites. More recent criteria for classification of primary gastrointestinal lymphoma includes cases where bulk of tumor involves GIT. ${ }^{[6,7]}$

\begin{tabular}{|l|l|}
\hline \multicolumn{3}{c}{ Access this article online } \\
\hline Quick Response Code: & Website: \\
\hline & www.jlponline.org \\
\hline
\end{tabular}

\section{CASE REPORT}

The blocks of an intestinal mass operated at a peripheral hospital were received at our institute for histomorphological and immunohistochemistry (IHC) confirmation. The following history is as per patient records.

A 30-year-old male presented to the surgical out-patient department of a peripheral hospital, with a recent history of abdominal distension, constant pain in the right lower quadrant along with nausea and vomiting. He was a known case of abdominal tuberculosis and had received a full course of anti-tubercular treatment. However, at the time of presentation, no relevant history of fever, night sweats, weight loss or bleeding per rectum was recorded. No evidence of superficial lymphadenopathy was seen. Hematological findings showed a total leucocyte count of $8900 / \mathrm{mm}^{3}$ and a differential count of neutrophils $68 \%$, lymphocytes $26 \%$, eosinophil 4\%, monocytes $2 \%$, and a hemoglobin of $11.3 \mathrm{~g} / \mathrm{dl}$. Barium study of the small intestine showed a stricture, at the terminal ileum. Computerized tomographic scan revealed a mass at the terminal ileum along with right sided pericolic infiltrate and mesenteric lymphadenitis. 
No evidence of hepatosplenomegaly was evident as per the records. Exploratory laparotomy was done to relieve patient symptoms and establish the diagnosis. Surgical intervention comprised of right hemicolectomy and ileocolic anastomoses. Histopathological examination report labeled it as suspicious of lymphoma.

Sections from the blocks received were evaluated for histomorphological and IHC categorization and confirmation. Microscopic examination of the lesion revealed multiple nodules of cellular infiltrates extending from intestinal mucosa up to the serosa. Cellular infiltrates comprised of mixed population of lymphocytes, plasma cells histiocytes, neutrophils, eosinophil, Reed-Sternberg (RS) cells and its variants [Figure 1]. Occasional mummified cells were also seen. IHC staining showed that the RS cells and its variants were positive for CD30 antigen [Figure 2] and weakly positive for CD15 and negative for CD45 [Figure 3]. In contrast, small lymphocytes in the background were positive for CD45. Stain for CD3 and CD20 showed normal pattern. On the basis of histomorphological features and IHC, a definitive diagnosis of HL, mixed cellularity type was established. A full work-up of patient to identify the presence of the disease anywhere else in the body was done. All investigations were within the normal limits hence a diagnosis of primary HL of the GIT was made.

\section{DISCUSSION}

Primary classical HL are well-established neoplasms of the lymphnode; however, the primary extranodal forms are rare and account for less than 1\%, GIT being the most common site. ${ }^{[1]}$ In view of its rare occurrence, diagnosis has to be made in accordance with histological as well as other clinical and radiological criteria. ${ }^{[6]}$ These can be outlined as: (a) no superficial lymphadenopathy should be present at the time of diagnosis (b) no involvement of mediastinal lymph node on chest imaging (c) complete blood count and WBC differential should be within normal limits (d) predominance of GIT lesion with or without positive adjacent lymph node (e) liver and spleen should be disease free at the time of diagnosis. The present case fulfilled all the criteria and was thus categorized as primary gastrointestinal HL.

The clinical manifestations of primary GIT lymphoma are limited. A case has been reported to be associated with cholelithiasis and ileocaecal intussusception, ${ }^{[2]}$ ileum being the most common site. It has been well-documented that ileum is rich in lymphoid follicles ${ }^{[4]}$ hence, neoplastic

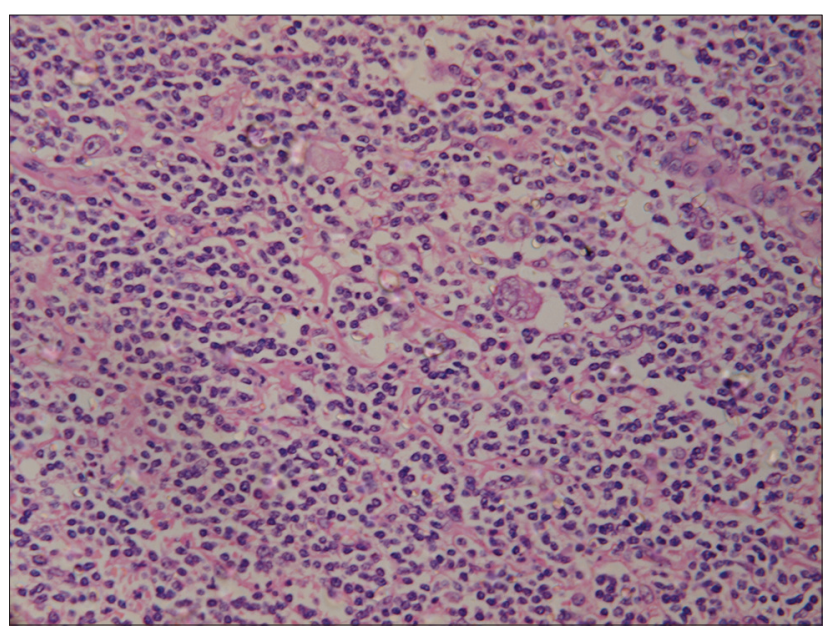

Figure 1: Photomicrograph of the lesion showing Reed-Sternberg cells in a polymorphous background $(\mathrm{H}$ and $\mathrm{E}, \times 40)$

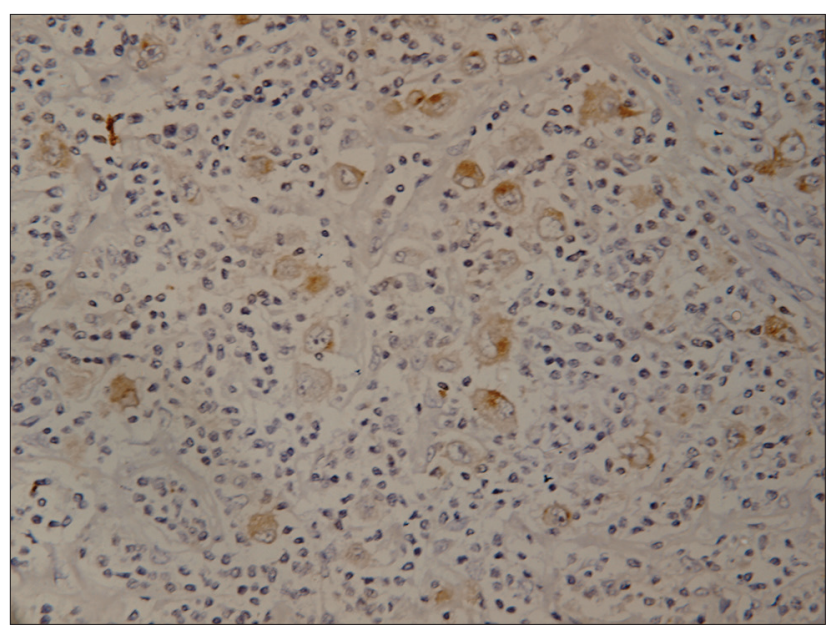

Figure 2: Photomicrograph showing Reed-Sternberg cells and its variants with CD30 positivity (Immunohistochemistry, $\times 40$ )

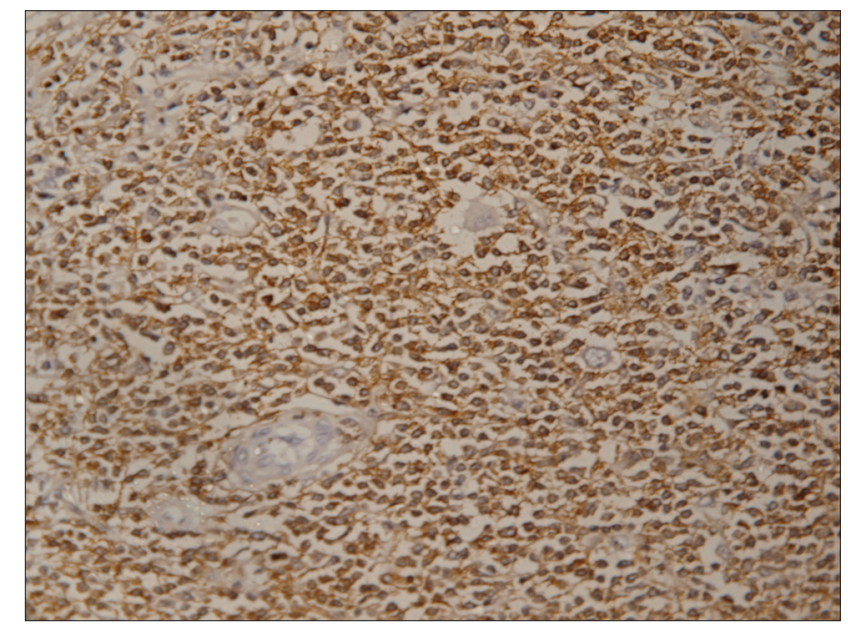

Figure 3: Photomicrograph showing CD45 negative Reed-Sternberg cells and its variants (Immunohistochemistry, $\times 40$ )

transformation due to changes in immune response can occur. ${ }^{[1]}$ Because it is rare for HL to localize to the small 
intestine, any diagnosis of the small bowel HL should prompt a thorough search for additional disease at the other site. ${ }^{[8]}$

HL presenting with primary bowel involvement is a unique clinical entity, despite the frequent inclusion of $\mathrm{HL}$ in various series of malignant lymphoma, reports on primary lesion of the small intestine are rare. Very few cases have been documented on review of the literature. A series of literature revealed only seven cases of intestinal HL out of 778 GIT lymphomas. ${ }^{[8]}$ In a case series, out of 24 cases of lymphoma of the GIT five cases of HL were reported. ${ }^{\left[{ }^{[]}\right.}$However, only two cases of primary HL were encountered out of a total of 117 patients who presented with gastrointestinal lymphoma. ${ }^{[10]}$ Only 11 individual patients with small intestine HL were recorded since 1967. ${ }^{[8]}$ However, their diagnosis was in doubt due to lack of IHC staining. ${ }^{[11]}$ Many of these patients were thought to resemble HL histologically such as anaplastic large cell lymphoma shows atypical cells resembling RS cells, similarly a highly differentiated carcinoma or multiple myeloma could also mimic HL with large RS like cells in a background of inflammatory cells. In the present case, the large atypical cells were negative for activin receptor-like kinase-1 ruling out anaplastic large cell lymphoma. Emphasis has to be laid on the importance of IHC. With currently available IHC techniques these histologically similar tumors can be readily differentiated from HL. ${ }^{[12]}$ In the present case, the IHC confirmed the histopathological presence of HL.

\section{CONCLUSION}

To conclude, since it is rare for HL to localize in the small intestine without involvement of regional or distant lymph nodes, any diagnosis of small bowel HL should prompt a thorough search for additional disease at other sites. Histological confirmation by IHC technique supports the diagnosis of small intestinal HL to be valid. All the criteria for inclusion should be adhered to for a conclusive diagnosis of a primary HL of the intestine.

\section{REFERENCES}

1. Gandhi JS, Mehta A, Sharma A, Kamboj M. Primary Hodgkin lymphoma of the ileum. J Cancer Res Ther 2010;6:342-3.

2. Haque MM, Siddique AB, Badruddoza SM, Manik MA. Primary intestinal Hodgkins lymphoma associated with intussuception and cholelithiasis. TAJ 2006;19:81-3.

3. Crowley KS, Don G, Gibson GE, Juttner CA, Miliauskas JR. Primary gastrointestinal tract lymphoma: A clinico-pathological study of 28 cases. Aust N Z J Med 1982;12:135-42.

4. Nguyen AT, Hashemi S, Castellano MR, Eisenbraun M, Coppa GF. A new subtype of Hodgkin's lymphoma, syncytial nodular sclerosing: First case report of primary small bowel lymphoma. J Gastrointest Cancer 2009;40:38-40.

5. Li S, Borowitz MJ. Primary Epstein-Barr virus-associated Hodgkin disease of the ileum complicating Crohn disease. Arch Pathol Lab Med 2001;125:424-7.

6. Dawson IM, Cornes JS, Morson BC. Primary malignant lymphoid tumours of the intestinal tract. Report of 37 cases with a study of factors influencing prognosis. Br J Surg 1961;49:80-9.

7. d'Amore F, Brincker H, Grønbaek K, Thorling K, Pedersen M, Jensen MK, et al. Non-Hodgkin's lymphoma of the gastrointestinal tract: A population-based analysis of incidence, geographic distribution, clinicopathologic presentation features, and prognosis. Danish lymphoma study group. J Clin Oncol 1994;12:1673-84.

8. Morgan PB, Kessel IL, Xiao SY, Colman M. Uncommon presentations of Hodgkin's disease. Case 1. Hodgkin's disease of the jejunum. J Clin Oncol 2004;22:193-5.

9. Maheshwari V, Tyagi SP, Aziz M, Ashraf SM, Hameed F, Alam K. Primary malignant lymphoma of the gastro-intestinal tract: A clinicopathological study of 24 cases. J Indian Med Assoc 1992;90:233-5.

10. Lewin KJ, Ranchod M, Dorfman RF. Lymphomas of the gastrointestinal tract: A study of 117 cases presenting with gastrointestinal disease. Cancer 1978;42:693-707.

11. Pai SA, Naresh KN, Borges AM. Primary Hodgkin's disease of the small intestine: Does it really exist? Indian J Gastroenterol 1994;13:150.

12. Fenoglio-Preiser CM, Noffsinger AE, Stemmermann GN, Lantz PE, Listron MB, Rilke FO. Gastrointestinal hematological lesions. In: Patterson AS, Iannuzzi M, Carita L, editors. Gastrointestinal Pathology: An Atlas and Text. Philadelphia, PA: Lippincott-Raven; 1999. p. 1129-68.

How to cite this article: Sharma S, Rana S, Kapur S, Jairajpuri ZS. Primary intestinal hodgkin's lymphoma: An uncommon presentation. J Lab Physicians 2013;5:124-6.

Source of Support: Nil. Conflict of Interest: None declared. 